\title{
Three-Year Follow-Up of Diastema Closure Using Ceramic Veneers: A Case Report
}

\section{AlHanouf A AlHabdan}

Department of Restorative Dental Sciences, King Saud University, Riyadh, KSA

*Corresponding author: AlHanouf A AlHabdan, BDS, MSc, Lecturer, Department of Restorative Dental Sciences, King Saud University, Riyadh, KSA, Tel: +966 11467 0000; E-mail: Alhanouf@habdan.com

Received date: June 15, 2017; Accepted date: June 23, 2017; Published date: July 01, 2017

Copyright: (c) 2017 AlHabdan AA.This is an open-access article distributed under the terms of the Creative Commons Attribution License, which permits unrestricted use, distribution, and reproduction in any medium, provided the original author and source are credited.

\begin{abstract}
The use of ceramic veneers has increased due to the minimal tooth preparation involved, the color and contour stability of the material, and the enhanced esthetics. They have proven to be an effective treatment option for managing esthetic and functional problems, especially in the anterior teeth. This paper aims to describe the esthetic management of anterior midline diastema and spacing in a 24-year-old female patient, using ceramic veneers.
\end{abstract}

Keywords Veneers; Ceramic; Diastema; Spaces; Esthetics

\section{Background}

Currently, the demand for dental esthetics and beautiful smiles has increased. Thus, dental practitioners should be able to provide adequate treatment planning and patient selection to achieve maximum esthetic and function [1]. Since the introduction of ceramic veneers in 1983 [2], veneers have evolved and undergone vast improvement, making it one of the most viable treatment modalities that can replace bleaching and enamel microabrasion. It is considered one of the most conservative operative treatment procedures [3] because the tooth preparation involves the removal of less than half the thickness of the enamel, leaving the remaining portion intact before veneer placement [2].

The use of ceramic veneers has increased due to color and contour stability, superior esthetics, and the involvement of minimal tooth preparation [4]. They have proven to be an effective treatment option to manage the esthetic and functional problems, especially in the anterior region [5]. Moreover, veneers are known to provide predictable, stable, and satisfactory results for both the patient and the dentist [6].

Owing to the various applications of ceramics in dentistry, several dental ceramics with varying mechanical properties and chemical composition have been introduced [7]. They are broadly categorized into glass and high-strength core ceramics, onto which an esthetic ceramic layer must be applied to achieve a natural appearance [8-10]. Lithium disilicates are glass ceramics that are currently recommended for porcelain laminate veneers (PLVs) due to the ability to produce thin layers, thereby making them more translucent and esthetic [11].

Ceramic veneers are indicated in cases of anterior dental wear, discoloration, minor changes in teeth contour, diastema closure, and sufficient remaining sound tooth structure $[5,12]$. The present study aimed to describe a method for the esthetic management of anterior midline diastema and spacing using ceramic veneers.

\section{Case Description}

\section{Diagnosis and treatment planning}

A 24-year-old female patient presented to the restorative clinics at the College of Dentistry, King Saud University, Saudi Arabia. She expressed her desire to complete her restorative treatment and find a long-term treatment for the gaps between her front teeth. The patient was unhappy with her smile and, therefore, avoided smiling. She reported a failed orthodontic treatment six years ago that ended up with a broken fixed retainer on the lingual surface of the maxillary anterior teeth.

Oral examination revealed the presence of diastemas between the maxillary anterior teeth (Figure 1)

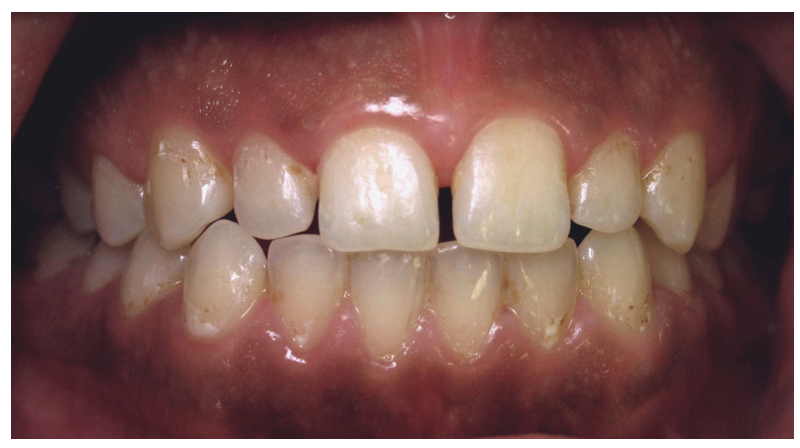

Figure 1: Preoperative view of maxillary anterior teeth in occlusion.

with multiple carious lesions and defective restorations in the posterior teeth and the facial surface of the lower anterior teeth. The patient has a class I molar-canine relation with $1-2 \mathrm{~mm}$ overjet, $1 \mathrm{~mm}$ overbite, and 1-2 mm diastema between the anterior teeth (Figures 2 and 3). 


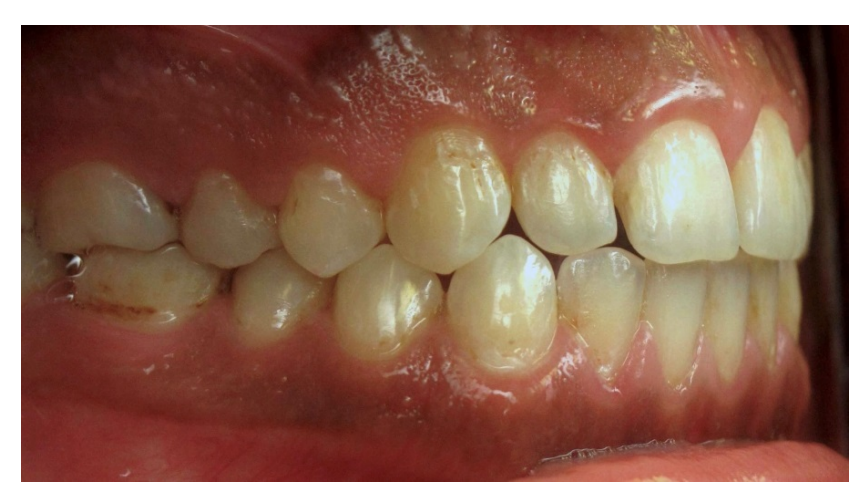

Figure 2: Preoperative view of right anterior side.

After thorough examination, complete radiographic and photographic data was collected from the patient. Impressions for diagnostic models were prepared with irreversible hydrocolloid (Heraplast, Heraeus Kulzer, USA) using type IV dental stone, and mounted on a semi-adjustable articulator. The models were studied to decide the shape and size of the restorations with the help of a diagnostic wax-up and mockup procedure.

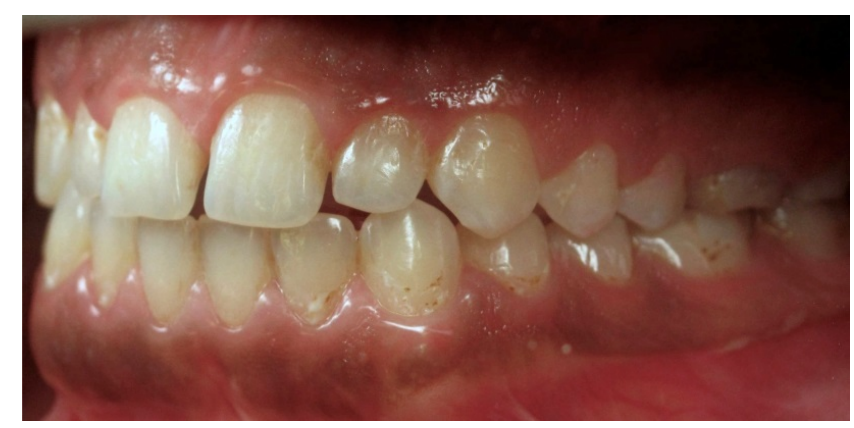

Figure 3: Preoperative view of left anterior side.

During the following appointment, the diagnostic wax-up was presented to the patient and any desired alteration by the patient was analyzed, discussed, and adjusted. To provide a long-term solution, the patient was provided with the option of ceramic veneers for the maxillary arch, whereas vital bleaching was suggested for the mandibular arch because they are less visible. The final treatment plan was approved after form and function were evaluated.

\section{Dental preparation}

Restorative treatment was performed on all the mandibular teeth. Then, the patient received in-office bleaching using 35\% hydrogen peroxide (Zoom! whitespeed, Philips-USA) followed by at-home bleaching using 15\% carbamide peroxide (opalescence PF 15\%, Ultradent Products, Inc., South Jordan UT, USA) and bleaching trays. After two weeks, the patient was evaluated for shade changes. Prior to the in-office treatment, she required an A3 shade, which changed to A1 at the end of the bleaching session. The shade was determined using Vitapan Classical shade guide (Vita Zahnfabrik, Germany).
On a subsequent appointment 2 weeks later, six maxillary anterior teeth were prepared to receive ceramic laminate veneers. The tooth preparation was performed at an enamel depth of 0.5-0.7 mm with 1.5 $\mathrm{mm}$ incisal reduction using a depth cutting tapered diamond bur (diameter, $1 \mathrm{~mm}$ ). Chamfer finish line was maintained in the cervical region (Figure 4) at the level of the gingival margin.

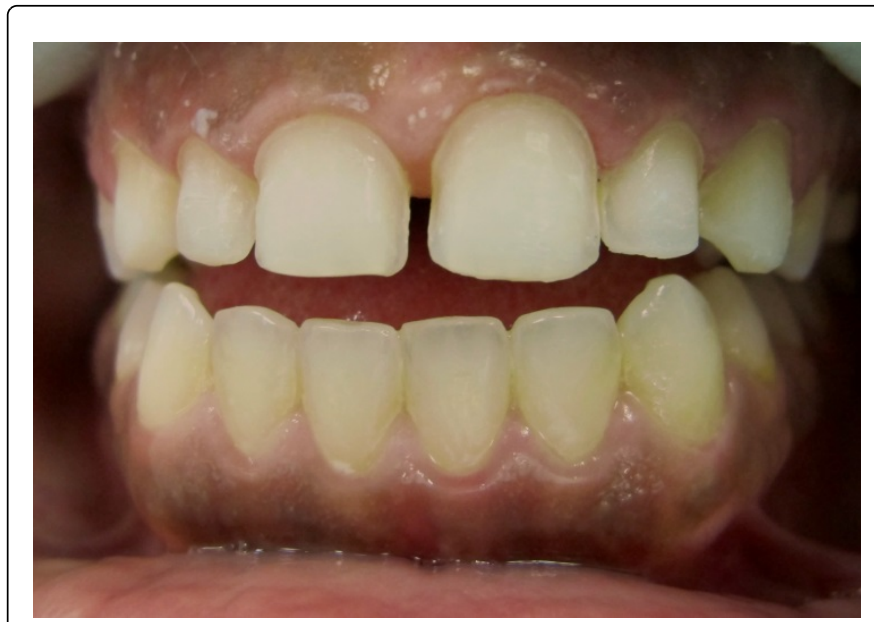

Figure 4: Anterior teeth after veneer preparation.

The proximal preparation was extended beyond the contact area on the mesial surface of the central incisors to avoid the visibility of the tooth restoration junction and the appearance of a black triangle.

\section{Chairside impression}

Gingival retraction was performed after finishing the sharp line and point angles (Ultrapak Cord \#000, Ultradent Products Inc., South Jordan, UT, USA) in order to provide for gingival sulcus enlargement without using impregnated cords with hemostatic or astringent solutions. Impressions were taken using a vinyl polysiloxane material (Express XT, 3M ESPE, Seefeld, Germany); the light-bodied impression material was simultaneously spread on the teeth and gently blown over the preparation while the heavy-bodied impression was loaded in the plastic tray and inserted in the oral cavity. The impression material was allowed to set according to manufacturer's instructions and then removed.

\section{Laboratory fabrication of veneers and cementation}

After one week, the patient returned for placement of the final veneers. Ceramic laminate veneer restorations were fabricated with a lithium disilicate-reinforced glass ceramic material (IPS e.max Press, IvoclarVivadent, Liechtenstein) using the heat press technique.

The prepared teeth were cleaned and the veneers were tried-in using a transparent try-in paste (Variolink Veneer try-in paste, Ivoclar). The margins of the veneers were checked for proper adaptation and patient satisfaction. Then, the internal surfaces of the veneers were cleaned and etched with hydrofluoric acid (ceramic Etchant 9,5\%, Bisco Inc., Schaumburg, IL, USA) for one min, washed under running water for another minute, and dried with an air syringe. Monobond-plus (Ivoclar Vivadent, Schaan, Lichtenstein) was applied and left to react for $60 \mathrm{~s}$ before air drying. 
The teeth surfaces were etched using $37 \%$ phosphoric acid for $30 \mathrm{~s}$, washed for another $30 \mathrm{~s}$ and gently dried. ExciTE F (Ivoclar Vivadent, Schaan, Lichtenstein) bonding agent was applied for $10 \mathrm{~s}$ using a microbrush. Excess bonding was blown to a thin film using air spray. Heliobond (Ivoclar Vivadent, Schaan, Lichtenstein) was directly applied on to the surfaces of the tooth and the veneers before cement application with a microbrush.

Light-cured resin cement (Variolink Veners, transparent shade, Ivoclar Vivadent, Lichtenstein) was applied to the inner surface of the veneers, which were gently seated on to their respective preparations using finger pressure. Excess cement was carefully removed with an explorer and a microbrush. Light polymerization was performed with a LED-curing unit (Blue phase LED-curing light, Ivoclar Vivadent) for $30 \mathrm{~s}$ from the buccal, incisal, mesial, and distal aspects of each tooth.

Residual cement was removed using a \#12 surgical blade and explorer. Flossing was performed at the proximal areas to ensure interproximal contact patency. The margins were polished with a silicone instrument $\mathrm{Pogo}^{\circ}$ (Dentsply/Caulk, Milford, DE, USA). Finally, the restorations were checked for any occlusal interference and the entire cementation procedure was performed one-by-one starting with the central incisors, laterals, and finally, the canines. Images of the final restorations are shown in Figure 5.

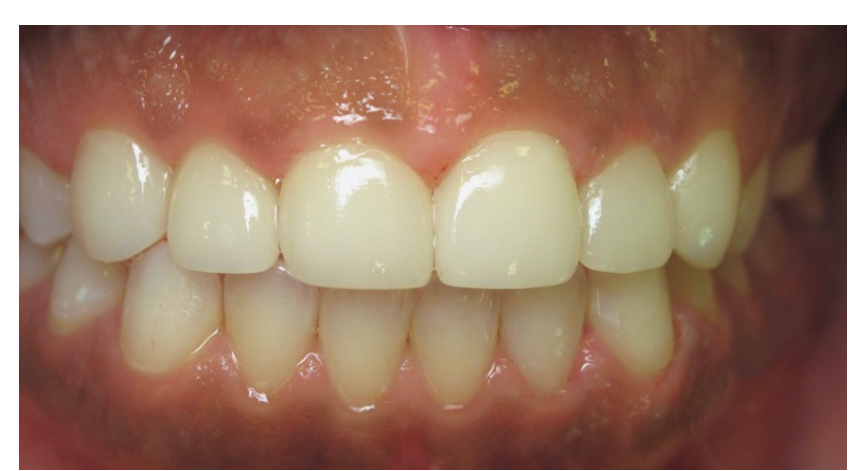

Figure 5: Immediate frontal view after cementation of the veneers.

\section{Esthetic result}

The patient was recalled for follow-up one week later. Adequate gingival health and patient satisfaction confirmed the success of this treatment.

A three-year follow-up asserted complete patient satisfaction with regard to her smile. No signs or symptoms of teeth sensitivity were observed, and gingival health was excellent (Figure 6).

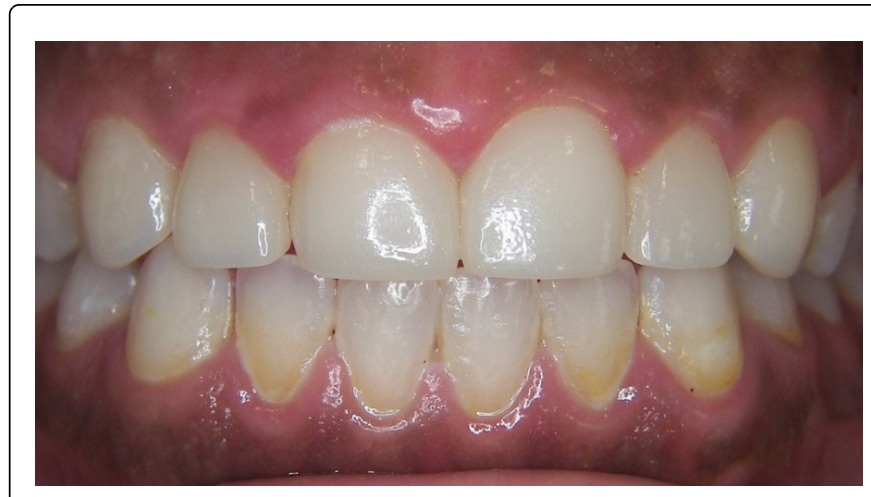

Figure 6: Three-year follow-up.

\section{Discussion}

The closure of diastemas is recognized as one of the most common and challenging tasks in restorative dentistry [13]. Diastemas can occur during normal dental development starting from the mixed dentition period [14]. The continuous appearance of diastemas between maxillary anterior teeth has been associated with both esthetics and malocclusion [15]. Anterior diastema is defined as "anterior midline spacing greater than $0.5 \mathrm{~mm}$ between the proximal surface of adjacent anterior teeth" [16].

The first line of treatment for diastemas is to ascertain the cause. Several aspects such as genetic and physiologic factors, abnormal frenum, abnormal habits, supernumerary teeth, and iatrogenic factors can affect the occurrence and size of an anterior diastema $[17,18]$. Proper examination and patient selection, accurate diagnosis and effective oral hygiene using a range of restorative and orthodontic methods are required for the effective treatment of anterior diastemas $[15,19]$.

Recently, ceramic veneers have been widely and effectively used to treat diastemas, with high esthetic results [20]. Adult patients may refuse to opt for orthodontic treatment because of the lengthy process and the need for immediate esthetic results. In the present case, the patient had undergone orthodontic treatment for a long period, and had expressed her dissatisfaction with the outcome. Her main request was the closure of the diastemas in a short time with long-term esthetic results. Thus, ceramic veneers were offered as the first treatment option. Ceramic veneers require minor tooth preparation; the use of these veneers is considered as a conservative treatment option when compared with all-ceramic crowns [21], which also produce high esthetic results, but involve extensive tooth reduction $(1.5-2 \mathrm{~mm})$ [2].

\section{Conclusion}

Ceramic veneers may prove to be effective in closing diastemas and should therefore, be considered as one of the main options during treatment planning.

\section{References}

1. Morita RK (2016) Minimally Invasive Laminate Veneers: Clinical Aspects in Treatment Planning and Cementation Procedures. Case Rep Dent 2016: 1839793. 
Citation: AlHanouf AA (2017) Three-Year Follow-Up of Diastema Closure Using Ceramic Veneers: A Case Report. Dentistry 7: 440. doi: $10.4172 / 2161-1122.1000440$

Page 4 of 4

2. Pini NP (2012) Advances in dental veneers: materials, applications, and techniques. Clin Cosmet Investig Dent 4: 9-16.

3. Heffernan MJ (2002) Relative translucency of six all-ceramic systems. Part II: core and veneer materials. J Prosthet Dent 88: 10-15.

4. Rotoli BT (2013) Porcelain veneers as an alternative for esthetic treatment: clinical report. Oper Dent 38: 459-466.

5. Kamble VD, Parkhedkar RD (2013) Esthetic rehabilitation of discolored anterior teeth with porcelain veneers. Contemp Clin Dent 4: 124-126.

6. Radz GM (2011) Minimum thickness anterior porcelain restorations. Dent Clin North Am 55: 353-370.

7. Hahnel S (2009) Surface characterization of dental ceramics and initial streptococcal adhesion in vitro. Dent Mater 25: 969-975.

8. Spear F, Holloway J (2008) Which all-ceramic system is optimal for anterior esthetics? J Am Dent Assoc 139: 19-24.

9. Denry I, Holloway JA (2010) Ceramics for Dental Applications: A Review. Materials 3: 351-368.

10. Tang X (2012) Effects of ageing on surface textures of veneering ceramics for zirconia frameworks. J Dent 40: 913-920.

11. Shenoy A, Shenoy N (2013) Dental ceramics: An update. J Conserv Dent 13: 195-203.

12. Cunha LFD (2013) Ceramic veneers with minimum preparation. Eur J Dent 7: 492-496.

13. Moon JE (2010) Esthetic restorations of maxillary anterior teeth with orthodontic treatment and porcelain laminate veneers: a case report. J Adv Prosthodont 2: 61-63.
14. Abraham R, Kamath G (2014) Midline diastema and its aetiology-a review. Dent Update 41: 462-464.

15. Huang WJ, Creath CJ (1995) The midline diastema: a review of its etiology and treatment. Pediatr Dent 17:171-179.

16. Keene HJ (1963) Distribution of diastemas in the dentition of man. Am J Phys Anthropol 21: 437-441.

17. Tanaka OM (2015) When the Midline Diastema Is Not Characteristic of the "Ugly Duckling" Stage. Case Rep Dent 2015: 924743.

18. Jaija AM, El-Beialy AR, Mostafa YA (2016) Revisiting the Factors Underlying Maxillary Midline Diastema. Scientifica 2016: 5607594.

19. Chu CH, Zhang CF, Jin LJ (2011) Treating a maxillary midline diastema in adult patients: a general dentist's perspective. J Am Dent Assoc 142: 1258-1264.

20. Viswambaran M, Londhe SM, Kumar V (2015) Conservative and esthetic management of diastema closure using porcelain laminate veneers. Med J Armed Forces India 71: 581-585.

21. Bayramoglu E, Ozkan YK, Yildiz C (2015) Comparison of marginal and internal fit of press-on-metal and conventional ceramic systems for threeand four-unit implant-supported partial fixed dental prostheses: An in vitro study. J Prosthet Dent 114: 52-58. 